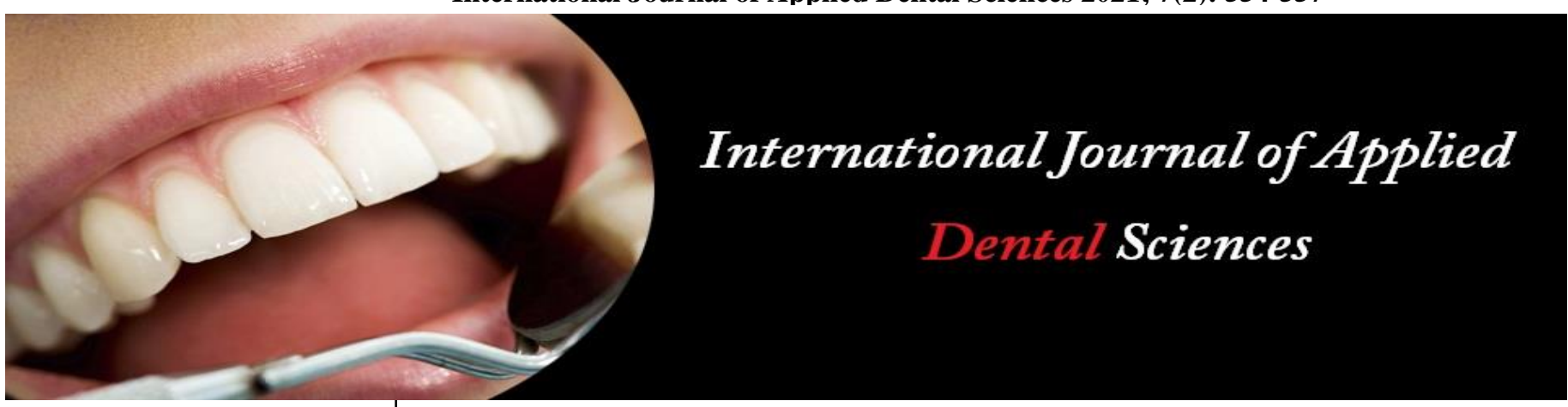

ISSN Print: 2394-7489

ISSN Online: 2394-7497

IJADS 2021; 7(2): 554-557

(C) 2021 IJADS

www.oraljournal.com

Received: 01-02-2021

Accepted: 03-03-2021

Dr. Anagha Sunder Kakapoyi Senior Resident, MGV's KBH Dental College and Hospital Nashik, Mumbai, Maharashtra. India

Dr. Seema Pattanaik

Professor, MGV's KBH Dental

College and Hospital Nashik,

Mumbai, Maharashtra, India

Dr. Bikash Pattanaik

Professor and Head, MGV's

KBH Dental College and

Hospital Nashik, Mumbai,

Maharashtra, India

Dr. Najma Shaikh

Senior Resident, MGV's KBH

Dental College and Hospital

Nashik, Mumbai, Maharashtra,

India

\section{Digital scanners in prosthodontics: A literature review}

\author{
Dr. Anagha Sunder Kakapoyi, Dr. Seema Pattanaik, Dr. Bikash Pattanaik \\ and Dr. Najma Shaikh
}

DOI: https://doi.org/10.22271/oral.2021.v7.i2i.1261

\section{Abstract}

Digital dentistry depends on computer-aided design (CAD) software and computer-aided manufacturing (CAM) systems. With CAD/CAM systems, a large quantity of desired tools for dentistry can be easily created with a high degree of accuracy. Digital scanning is one of the recent method used in dentistry. It provides accuracy, precisions to the prosthesis and it also as more advantages as compared to conventional techniques. Aim of this publication is to provide an extensive review on intra-oral scanners used in dentistry with particular attention given to the history, working principle of scanners, knowledge about accuracy and precision of all the scanners.

Keywords: digital scanners, digital impressions, CAD-CAM, digital dentistry, accuracy

\section{Introduction}

In current dental practice, a large number of digital technology is available. To improve diagnostics, treatment planning, increase accuracy,efficiency and reduce costs.In dentistry world, three dimensional (3D) technology is rapidly entering.

Precision and accuracy of master impressions are critical and an important factor to the overall excellence and marginal fit of definitive fixed restorations. CAD/CAM make the work of clinicians, patients and laboratory technician's easier, reproducible and accurate, and allows for user and patient friendly clinical procedures. CAD/CAM systems are available in different types either it can be digitally scanned and create fixed restorations, chairside or that capture chairside digital impressions that are then sent to a laboratory. In-office CAD/CAM allows clinicians to provide indirect fixed restorations in the same visit that are accurate and esthetically pleasing.

\section{Components of CAD/CAM are}

Scanner/tool will transforms geometry into digital data which can be processed by the computer ${ }^{[1]}$.

Software that processes data and, depending on the application, produces a data set for the product to be fabricated ${ }^{[1]}$.

A production technology that transforms the data set into the desired product ${ }^{[1]}$.

\section{Advantages}

Accuracy: trueness and precision is more when we use digital impression. It has more accurate marginal and internal fit.

Patients Outcome: Digital impression was more comfortable to the patient as there were no gag reflex, taste irritation, anxiety, difficulty to breathe. Patient's preference was more for digital impression, as they were more concern on comfort.

Operator Outcome: The work flow of digital impression technique took less time. Missing and unacceptable area can be corrected more easily with digital impression. Risk of contamination also reduced ${ }^{[3,4]}$.

\section{Corresponding Author:} Dr. Anagha Sunder Kakapoyi Senior Resident, MGV's KBH Dental College and Hospital Nashik, Mumbai, Maharashtra, India 


\section{Limitations}

1. When multiple unit bridge are fabricated, we must be concerned about the passive fit and possibility of repair.

2. In long extended bridges which are milled in one piece, it can lead to strain development mostly in angled type bridges.

3. In veneer technique also there can be chances of strain development.

4. There are limitation while capturing multiple element prosthesis like in cases of full mouth rehabilitation

5. Limitation of cost and accessibility.

6. All the systems and restorative materials for crowns,bridges,removable partial denture and complete denture prosthesis are not available in all the countries.

7. Proper investigation of the software and material is required before making the choices for clinical cases ${ }^{[5]}$.

\section{Back to the Past}

1973: Dr. Duret first introduced the CAD/CAM concept to dentistry. Dr. Mormann further developed the Concept of CAD/CAM systems.

1980: CEREC was the first digital impression system for use in the field of dentistry by Mormann Dr. Anderson developed Procera System.

1987: The CEREC 1 system (Sirona, Bensheim, Germany) together with the Duret system as the first intraoral digital impression

1998: DirectScan by HINT - ELS GMBH (DE)

2007: Cadent Inc (Carstadt, NJ) introduced iTero to the market

2008: Lava TM C.O.S. (Lava Chairside Oral Scanner; 3M

ESPE, Seefeld, Germany)

2011: TRIOS, was introduced by 3Shape (Copenhagen, Denmark)

2012: latest CEREC AC Omnicam, was brought to market ${ }^{[1}$, 2].

\section{Review of Literature \\ Digital Systems Used for Impression CEREC System}

The CEREC system stands for Chairside Economical Restoration of Esthetic Ceramic. CEREC 1 is a 2D system which can fabricate inlay for immediate cementation. CEREC 3 was used for inlays, onlays, veneers, crowns as 3 unit bridges \& crowns ${ }^{[6,1]}$.

\section{CEREC AC Bluecam}

It is the Fourth generation system. Currently it is the most prevalent CEREC system out of all the CEREC. Light source which is emitted was LED blue diode. It can capture one quadrant within 1 minute and the antagonist in a few seconds. Bluecam can be used for single tooth ${ }^{[6,1]}$

\section{CEREC Omnicam}

Latest Omnicam takes continuous various images, where a 3D model is generated after data acquisition. Omnicam can be used for a single tooth, quadrant, or full arch. Powder-free scanning and precise 3D images with natural color are the most prominent features of Omnicam ${ }^{[1,6]}$.

Principle: Triangulation measurement principle was used in CEREC System. It measures the angles and distances from known points with projected laser light. According to the Pythagorean theorem as light reflects off the object, the system determines the angle of reflection, and therefore the distance from the laser source to the object's surface, To provide uniform and predictable light dispersion, this technology requires a thin coating of opaque powder to be applied to the target tissue ${ }^{[1,6,7,9]}$.

\section{Direct Scan by HINT - ELS GMBH (DE)}

The first serial product of the Hint-ELs DentaCad System was introduced in 1998. The principle of the system is based on human stereoscopic vision and on the principle of the linear Projection.if straight lines are projected onto an object, the lines will be curved around the object. This distortion of the lines allows conclusions about the surface contour. The accuracy is in the range $12-15 \mu \mathrm{m}$, thus resulting more precise with respect to many of the 'popular' desktop scanning. The optical scanner takes a rapid sequence of pictures from different angles, every $200 \mathrm{~ms}$, it will record the surface and shape of every tooth or gap. Then the dentist, inputs the images into a 3D software, which conducts a pixelprecise comparison, and it maps the patient's mouth. The output data of the intra-oral scanner will be available in the standard STL file format and it can be processed by CAD/CAM components of Hint-ELs or by other open systems. Scanned data can also be automatically transferred with the help of the internet to a partner laboratory, equipped with a CAM machine. The design software includes a virtual articulator and allows the modelling of all anatomical inlays, crowns and large-span bridges ${ }^{[2,10]}$.

\section{Lava C.O.S. system}

It works on active wavefront sampling principle. It is a singlelens imaging system. Light source which is used is visible blue light. The Lava C.O.S. has the smallest scanner tip which is only $13.2-\mathrm{mm}$ wide. 3 sensors are used to capture image from different angulation, Simultaneously which develop surface patches with in focus in and out of focus data by Proprietary image processing algorithms.

\section{iTero system}

Coating of the teeth with scanning powder is not required in iTero system. Red laser is the light source in this system and it also consists of a host computer, a mouse, a keyboard, a screen, and a scanner. iTero is an open system for the treatment of single crowns, Fixed Partial Dentures, veneers, implants, aligners, and retainers. Digital image files are send in STL format, which can be shared to any other laboratory which is equipped with a CAD/CAM system.

Principle: Parallel confocal imaging projects laser light through a filtering pinhole to the target tissue The sensor is placed at the confocal (in-focus) imaging plane relative to the target. A small aperture in front of the sensor will block any light from above or below the plane of focus. Only the focused light which is reflecting off the target tissue will reenter the filter and reach the sensor for processing; out-offocus light (bad data) is eliminated, thus maximizing the accuracy of the scan. A parallel confocal system, tomographically slices the object and stitches together thousands of slices of data which will create a complete picture a process referred to as "point-and-stitch reconstruction" ${ }^{[8]}$.

\section{E4D system}

E4D System used the principle of optical coherence tomography and confocal microscopy ${ }^{[9]}$. Micro mirrors and red laser is used as a light source to vibrate 20,000 cycles per second. E4D's are having high-speed laser those formulates a 
digital impression of the prepared and proximal teeth such as to create an interactive 3 dimensional image. This system also functions as a powder-free intraoral scanning device. It includes a cart with the design center (computer and monitor), laser scanner head, and a separate milling unit. It is also said as single-visit treatment because in one appoinment we can do the digital scanning, designing the patterns, fabrication of prosthesis and prosthesis is delivered in single appointment. It provide high strength ceramic prostheses or composite even for minimally prepared teeth ${ }^{[1]}$.

\section{TRIOS system}

Principle used in TRIOS is Confocal microscopy ${ }^{[8]}$. Moreover, they have a quick scanning speed of up to 3000 images per second thereby reducing the influence of relative movement between scanner probe and teeth. Analyzing multiple number of pictures obtained, this system can create a final digital 3 dimensional model spontaneously to reflect the exact configuration of teeth and gingival color. Similar to the iTero and E4D systems, the TRIOS intra-oral scanner is a powder-free device in the scanning process.

TRIOS include two parts they are TRIOSR Cart and TRIOSR Pod. The TRIOSR Pod is having a hand held scanner which offers better flexibility and mobility, so due to its simple construction it is compatible with other computers and iPad also. ${ }^{1}$ Create high-quality digital impressions in life like colors and it activates quality dialogue with patients. Apply shade measurements to save time and create high quality resotrations. To get a perfect restorations TRIOS patient specific motion will let you record the patient's own movements highlighting the static and dynamic contact points.

\section{Discussion}

CAD/CAM in the field of dentistry have been around the early 1970's when the visionary, Dr werner Mormann developed CEREC. Since then its use in dentistry has been increasing day by day. Many companies are developing different software for quick and quality production of restorative prosthesis they are still working on the advancement of the software.

The definitive impression plays a critical role in the longevity of the prosthesis. There are many advantages of intraoralscanner over conventional technique making impression making.

Chandran $\mathrm{k}$ et al conducted a review, he stated that intra-oral digital scanners are superior to conventional impression technique ${ }^{[3]}$. Alikhazi $\mathrm{M}$ also et al suggested that outcome with digital impression is superior to conventional technique. ${ }^{9}$ Patzelt., et al. observed that digital impressions are efficient over time since they allow the reduction of work times, more convenient for the patient when compared with a conventional impression ${ }^{[11]}$. Mangano in his study, agreed that the Intra-oral scanner are not that effective in terms of complete arch when compared to conventional impression ${ }^{[12]}$. Cerec provided a chair-side system and a miling unit which allowed to produce highly esthetic prosthesis in single visit ${ }^{[13]}$. It has got a drawback by using an excessive coating of titanium dioxide during the scanning process, it can reduce the precision of marginal and internal fit ${ }^{[14.15]}$ Marignal fit errors which can be occurred due to powder scanning software can be minimized by using other scanners like itero, trios.

Many authors have done researches on intra-oral digital scanners regarding their accuracy trueness and precision on recording minute details of the soft and hard tissue when it comes to single prosthesis as well as full mouth prosthesis. Precision is seen more when done for single prosthesis or short arch prosthesis compared to full arch. Atieh., et al. in their study, established that intra-oral scanner were least accurate compared to conventional in full arch impression and it was adequate with quadrant arch ${ }^{[16]}$. Ryan Jin Young., et al. compared 9 intra-oral scanner to check the accuracy of full mouth image acquisition and stated that saliva, blood, moisture from the breath, tongue movement can also influence the performance of the scanners ${ }^{[16]}$.

Jung Lim et al conducted a study in which he compared trio and itero scanners and stated that the mean precision of the Trios scanner was greater than that of the iTero (marginal gap - Trios, $52.30 \mathrm{~mm}$; iTero, $60.46 \mathrm{~mm}$; P<.01). ${ }^{17}$ Hack and Patzelt compared Trios, ITero, Omnicam and Planscan and stated that more accuracy was with Trios and least with Omnicam and Planscan ${ }^{[18]}$. Guth et al stated In terms of trueness Cerec Bluecam and Omnicam were least accurate ${ }^{[19]}$. With respect to ease, trueness, accuracy and precision it is concluded that intra-oral digital scanner are superior when compared with conventional technology. Precision of the scanner still cannot be justified as most of the study were done in vitro. It has many limitation and error as the scanning is done on the master model. This leads us to believe that more in vivo study has to be done to assess the reliability of the intra oral scanners.

\section{Conclusion}

Digital Intra-oral scanner allows us to stay ahead of the dentistry world. It enhances patient experience and reduce chair time. It also reduce stress, simplifies the traditional work flow and achieve better communication with the laboratory. It has got some disadvantages as when it comes in contact with saliva, blood or movement with the patient. There is no scanner or technology that can now be considered more accurate due to the lack of standardized procedure. More clinical comparative studies as to be done regarding the accuracy and superiority of the scanners.

\section{References}

1. Yamini R, Parmar S, Abrol S, Nagpal A, Gupta R. Digital Impression : A New Era in Prosthodontics, IOSRJDMS 2017;16:82-84

2. Logozzo S, Franceschini G, Kilpelä A, Caponi M, Governi L, Blois L. A comparative analysis of intraoral 3D digital scanners for restorative dentistry, Internet $\mathbf{J}$ Med Technol 2011;5(1):1-2.

3. Chandran SK, Jaini J, Babu AS, Mathew A, Keepanasseril A. Digital Versus Conventional Impressions in Dentistry: A Systematic Review, Journal of Clinical \& Diagnostic Research 2019;13(4).

4. Mangano F, Gandolfi A, Luongo G, Logozzo S. Intraoral scanners in dentistry: a review of the current literature, BMC oral health 2017;17(1):149

5. Samra, Adriana, Morais, Eduardo, Mazur, Rui et al. CAD/CAM in dentistry - a critical review, Revista Odonto Ciência 2016;31:140. 10.15448/19806523.2016.3.21002.

6. Sannino G, Germano F, Arcuri L, Bigelli E, Arcuri C, Barlattani A. Cerec CAD/CAM chairside system, Oral \& implantology 2014;7(3):57.

7. Logozzo S, Zanetti EM, Franceschini G, Kilpelä A, Mäkynen A. Recent advances in dental optics-Part I: 3D intraoral scanners for restorative dentistry, Optics and 
Lasers in Engineering 2014;54:203-21.

8. Kravitz ND, Groth CH, Jones PE, Graham JW, Redmond WR. Intraoral digital scanners, J Clin Orthod 2014;48(6):337-47.

9. Alikhasi M, Siadat H, Nasirpour A, Hasanzade M. Threedimensional accuracy of digital impression versus conventional method: effect of implant angulation and connection Type, International journal of dentistry 2018.

10. Logozzo S, Zanetti EM, Franceschini G, Kilpelä A, Mäkynen A. Recent advances in dental optics-Part I: 3D intraoral scanners for restorative dentistry, Optics and Lasers in Engineering 2014;54:203-21.

11. Patzelt Sebastian, Emmanouilidi Archontia, Stampf Susanne, Strub Joerg, Att Wael. Accuracy of full-arch scans using intraoral scanners, Clinical oral investigations, 2013 18. 10.1007/s00784-013-1132-y.

12. Mangano F, Gandolfi A, Luongo G, Logozzo S. Intraoral scanners in dentistry: a review of the current literature, BMC Oral Health 2017;17(1):149. Published 2017 Dec 12.

13. Yuzbasioglu E, Kurt H, Turunc R, Biller H. Comparison of digital and conventional impression techniques: evaluation of patients' perception, treatment comfort, effectiveness and clinical outcomes, BMC Oral Health 2014;10.1186/1472-6831.

14. Hategan S, Gabor A, Zaharia C, Sinescu C, Negrutiu ML, Jivanescu A. Influence of scanning system and dentist's level of training in the accuracy of digital impressions, Proc. of SPIE 2016;9670:967014-1, doi: 10.1117/12.2189683.

15. Hategan SI, Ionel TF, Goguta L, Gavrilovici A, Negrutiu ML, Jivanescu A. Powder and powder-free intra-oral scanners: Digital impression accuracy, Primary dental journal 2018;7(2):40-3.

16. Atieh MA, Ritter AV, Ko CC, Duqum I. Accuracy evaluation of intraoral optical impressions: A clinical study using a reference appliance, The Journal of prosthetic dentistry 2017;118(3):400-5.

17. Lim JH, Park JM, Kim M, Heo SJ, Myung JY. Comparison of digital intraoral scanner reproducibility and image trueness considering repetitive experience, The Journal of prosthetic dentistry 2018;119(2):225-32.

18. Hack GD, Patzelt SB. Evaluation of the accuracy of six intraoral scanning devices: An in-vitro investigation, ADA Prof Prod Rev 2015;10:1-5.

19. Güth JF, Runkel C, Beuer F, Stimmelmayr M, Edelh off D, Keul C. Accuracy of five intraoral scanners compared to indirect digitalization, Clin Oral Investig 2017;21:1445-55 